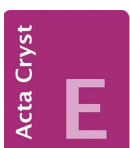

CRYSTALLOGRAPHIC COMMUNICATIONS

ISSN 2056-9890

Received 13 May 2020

Accepted 1 June 2020

Edited by M. Weil, Vienna University of Technology, Austria

Keywords: crystal structure; orthophosphate; cobalt; strontium; bifurcated hydrogen bonding; infrared spectroscopy.

CCDC reference: 2006988

Supporting information: this article has supporting information at journals.iucr.org/e

\section{Crystal structure of $\mathrm{SrCo}_{4}(\mathrm{OH})\left(\mathrm{PO}_{4}\right)_{3}$, a new hydroxyphosphate}

\author{
Fatima-Zahra Cherif, ${ }^{a *}$ Mhamed Taibi, ${ }^{\mathrm{b}}$ Ali Boukhari, ${ }^{\mathrm{a}}$ Jilali Aride, ${ }^{\mathrm{b}}$ Abderrazzak \\ Assani, ${ }^{a}$ Mohamed Saadi ${ }^{a}$ and Lahcen El Ammari ${ }^{a}$
}

\begin{abstract}
a Laboratoire de Chimie Appliquée des Matériaux, Centre des Sciences des Matériaux, Faculty of Science, Mohammed V University in Rabat, Avenue Ibn Batouta, BP 1014, Rabat, Morocco, and ${ }^{\mathbf{b}}$ Laboratoire de Physico-Chimie des Matériaux Inorganiques et Organiques, Centre des Sciences des Matériaux, Ecole Normale Supérieure, Mohammed V University in Rabat, Morocco. *Correspondence e-mail: fz.cherif@yahoo.com
\end{abstract}

Single crystals of strontium tetracobalt tris(orthophosphate) hydroxide, $\mathrm{SrCo}_{4}(\mathrm{OH})\left(\mathrm{PO}_{4}\right)_{3}$, were grown serendipitously under hydrothermal conditions at $473 \mathrm{~K}$. The crystal structure consists of undulating chains of edge-sharing $\left[\mathrm{CoO}_{6}\right]$ octahedra that are linked into (010) layers by common vertices between chains. Adjacent layers are linked along [010] into a framework structure by tetrahedral $\left[\mathrm{CoO}_{4}\right]$ units and by $\mathrm{PO}_{4}$ tetrahedra. The framework delimits channels extending along [100] in which the eleven-coordinate strontium cations are situated. Bifurcated $\mathrm{O}-\mathrm{H} \cdots \mathrm{O}$ hydrogen bonds of weak strengths consolidate the crystal packing. The title compound was also characterized by infrared spectroscopy.

\section{Chemical context}

The search for new inorganic materials with open-frame structures comprising transition-metal polyhedra, $\left[M \mathrm{O}_{x}\right]$, with tetrahedral phosphate or vanadate units by sharing corners or edges is still ongoing (Rghioui et al., 2019; Ouaatta et al., 2019; Khmiyas et al., 2020). Generally, these interconnections can lead to structures with cages, interlayer spaces or channels, and the corresponding compounds are explored extensively for their excellent physical properties and various applications in electrical, electrochemical, magnetic or catalytic processes (Goodenough et al., 1976; Borel et al., 1991; La Parola et al., 2018; Hadouchi et al., 2019). The introduction of borate groups $\left(\mathrm{BO}_{3}\right.$ or $\left.\mathrm{BO}_{4}\right)$ to phosphate $\left(\mathrm{PO}_{4}\right)$ units leads to a group of borophosphates with specific structural characteristics. Compounds of this family likewise exhibit remarkable physicochemical properties that allow them to be applied in different fields (Kniep et al., 1998; Ewald et al., 2007; Lin et al., 2008; Menezes et al., 2008). About a decade ago, we managed to synthesize two borophosphate phases, viz. $\left(\mathrm{Ag}_{0.57} \mathrm{Ni}_{0.22}\right)$ $\mathrm{Ni}\left(\mathrm{H}_{2} \mathrm{O}\right)_{2}\left[\mathrm{BP}_{2} \mathrm{O}_{8}\right] \cdot 0.67 \mathrm{H}_{2} \mathrm{O}$ and $\mathrm{AgMg}\left(\mathrm{H}_{2} \mathrm{O}\right)_{2}\left[\mathrm{BP}_{2} \mathrm{O}_{8}\right] \cdot \mathrm{H}_{2} \mathrm{O}$ (Zouihri et al., 2011a,b). In this context, we attempted to synthesize a strontium- and cobalt-based borophosphate, namely $\mathrm{SrCo}_{2} \mathrm{BPO}_{7}$, by means of the hydrothermal process. Instead, we have isolated a new hydroxyphosphate, $\mathrm{SrCo}_{4}(\mathrm{OH})\left(\mathrm{PO}_{4}\right)_{3}$, and report here its crystal structure and its infrared spectrum.

\section{Structural commentary}

In the three-dimensional framework structure of $\mathrm{SrCo}_{4}(\mathrm{OH})\left(\mathrm{PO}_{4}\right)_{3}$, an octahedral coordination of three cobalt 


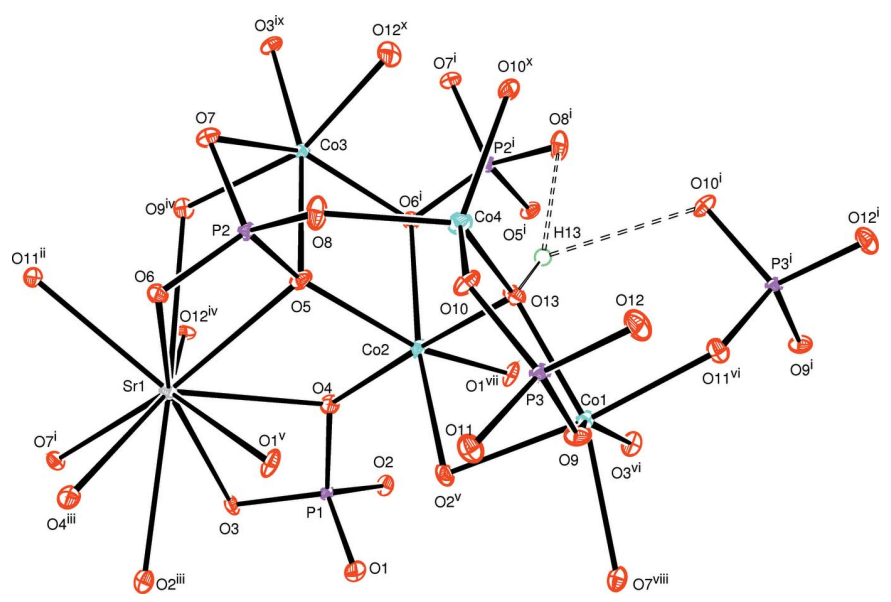

Figure 1

The interconnection of $\left[\mathrm{CoO}_{6}\right]$ and $\left[\mathrm{CoO}_{4}\right]$ polyhedra, and the hydroxide group linked to $\mathrm{PO}_{4}$ tetrahedra through a bifurcated hydrogen bond (dashed lines). Displacement ellipsoids are drawn at the $50 \%$ probability level. [Symmetry codes: (i) $-x, y+\frac{1}{2},-z+\frac{3}{2}$; (ii) $-x-\frac{1}{2},-y+1, z+\frac{1}{2}$; (iii) $x-1, y, z$; (iv) $-x+\frac{1}{2},-y+1, z+\frac{1}{2}$; (v) $x-\frac{1}{2},-y+\frac{3}{2},-z+1$; (vi) $x+1, y, z$; (vii) $x+\frac{1}{2},-y+\frac{3}{2},-z+1$; (viii) $-x+\frac{1}{2},-y+1, z-\frac{1}{2}$; (ix) $-x+1, y-\frac{1}{2}$, $\left.-z+\frac{3}{2} ;(x) x+\frac{1}{2},-y+\frac{1}{2},-z+1\right]$.

atoms $(\mathrm{Co} 1, \mathrm{Co} 2, \mathrm{Co} 3)$ and a tetrahedral coordination of the fourth cobalt $(\mathrm{Co} 4)$ is observed. Atom $\mathrm{O} 13$ bears a hydrogen atom and bridges two of the six-coordinate Co atoms (Co1, $\mathrm{Co} 2$ ) and the four-coordinate $\mathrm{Co} 4$ atom. The hydroxide group

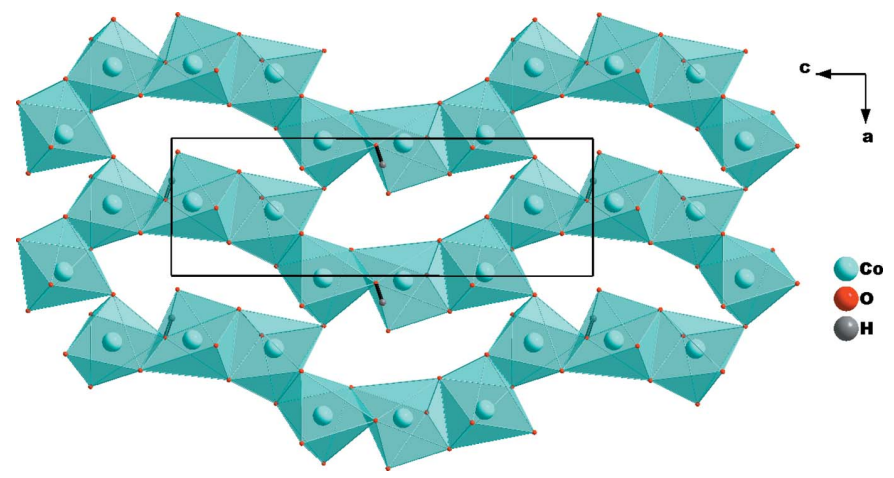

Figure 2

$\left[\mathrm{CoO}_{6}\right]$ octahedra sharing edges to form chains that are linked together via a common corner every three octahedra.

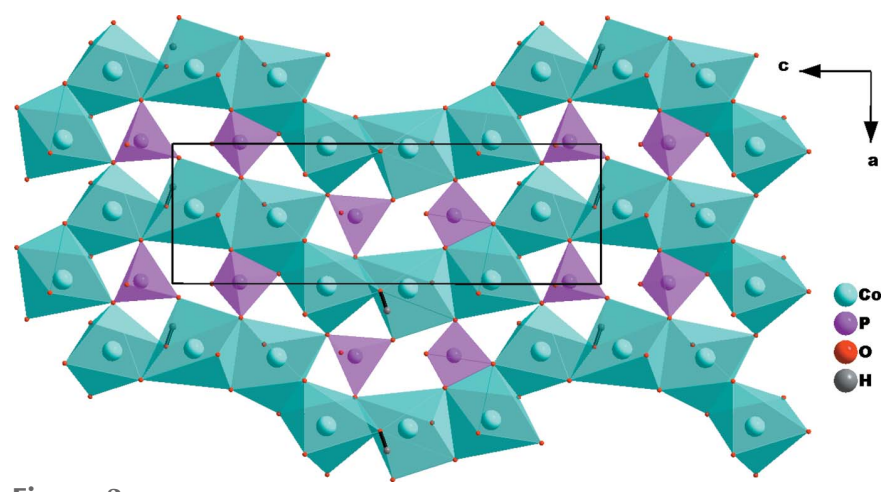

Figure 3

Edge-sharing $\left[\mathrm{CoO}_{6}\right]$ octahedra and $\mathrm{PO}_{4}$ tetrahedra building a layer parallel to $(010)$.
Table 1

Hydrogen-bond geometry $\left(\AA,^{\circ}\right)$.

\begin{tabular}{lllll}
\hline$D-\mathrm{H} \cdots A$ & $D-\mathrm{H}$ & $\mathrm{H} \cdots A$ & $D \cdots A$ & $D-\mathrm{H} \cdots A$ \\
\hline $\mathrm{O} 13-\mathrm{H} 13 \cdots \mathrm{O}^{\mathrm{i}}$ & 0.82 & 2.21 & $2.982(3)$ & 157 \\
$\mathrm{O} 13-\mathrm{H} 13 \cdots \mathrm{O} 10^{\mathrm{i}}$ & 0.82 & 2.40 & $3.010(3)$ & 132 \\
\hline
\end{tabular}

Symmetry code: (i) $x+1, y, z$.

also forms a weak bifurcated hydrogen bond (Table 1) to two phosphate tetrahedra (Fig. 1).

The $\left[\mathrm{CoO}_{6}\right]$ octahedra share edges to form infinite undulating chains extending parallel to [001]. Adjacent chains are cross-linked via common vertex atoms (O3) to build up (010) layers (Fig. 2) with the formation of oval voids surrounded by eight octahedra. Two $\mathrm{PO}_{4}$ tetrahedra occupy the void space, whereby $\mathrm{P} \mathrm{O}_{4}$ shares three of its vertices with five $\left[\mathrm{CoO}_{6}\right]$ octahedra and $\mathrm{P}_{2} \mathrm{O}_{4}$ shares an edge with an octahedron and a vertex with two opposite octahedra (Fig. 3).

The $\left[\mathrm{Co} 4 \mathrm{O}_{3} \mathrm{OH}\right]$ tetrahedra are linked through corners into zigzag chains running parallel to [100]; the chains are flanked by $\mathrm{P}_{2} \mathrm{O}_{4}$ and $\mathrm{P}_{3} \mathrm{O}_{4}$ tetrahedra into ribbons. The strontium cations and $\mathrm{P} \mathrm{O}_{4}$ tetrahedra are of the same height as the ribbons, thus defining a second layer parallel to (010) (Fig. 4).

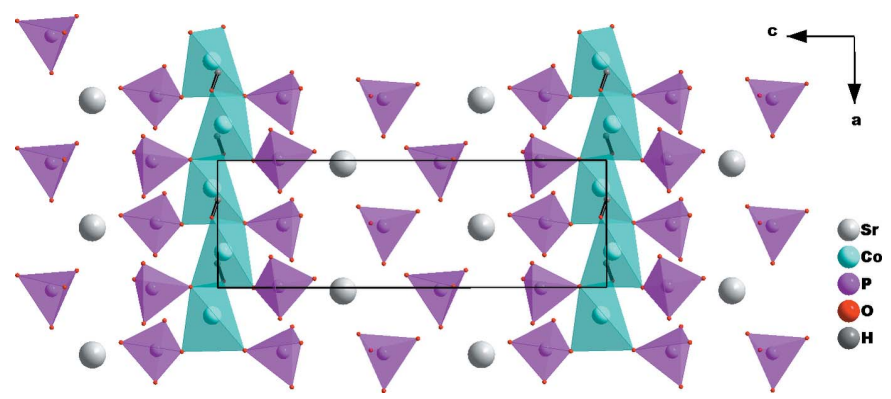

Figure 4

Ribbons formed by $\left[\mathrm{CoO}_{4}\right]$ and $\mathrm{P}_{2} \mathrm{O}_{4}$ and $\mathrm{P}_{3} \mathrm{O}_{4}$ tetrahedra, and strontium atoms and $\mathrm{P} \mathrm{O}_{4}$ tetrahedra at the same height forming the second layer parallel to (010).

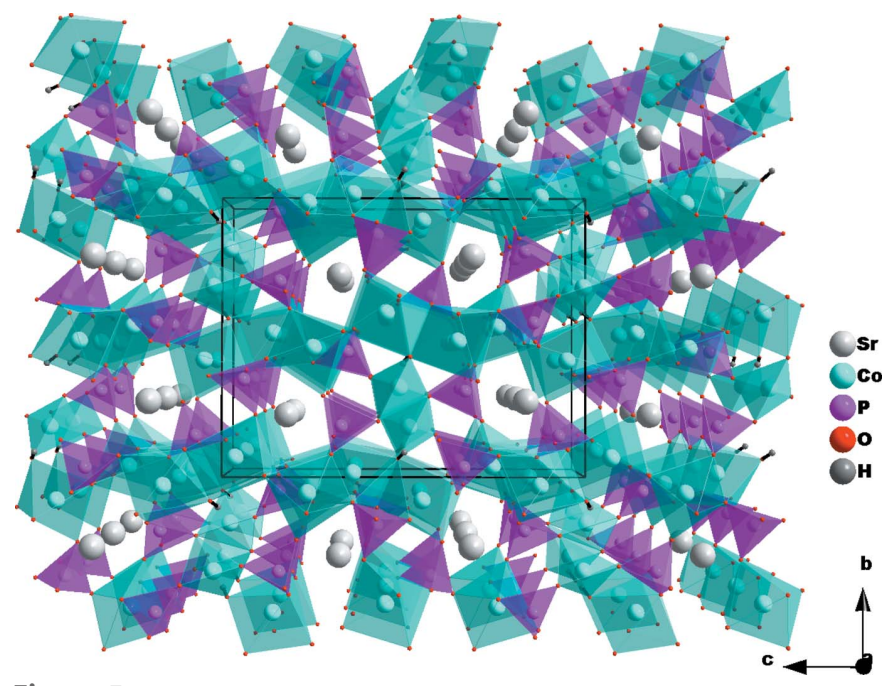

Figure 5

The crystal structure of $\mathrm{SrCo}_{4}(\mathrm{OH})\left(\mathrm{PO}_{4}\right)_{3}$ in a projection along [100], showing channels in which the strontium cations are located. 
The crystal structure can be described by the stacking of the two types of layers along [010], which leads to the formation of channels extending parallel to [100] in which the strontium cations are located (Fig. 5). Each $\mathrm{Sr}^{\mathrm{II}}$ atom is surrounded by eleven oxygen atoms, forming a distorted polyhedron.

Comparison of the metal-oxygen polyhedra in the title structure with the same type of polyhedra in comparable structures shows a similar behaviour. All $\left[\mathrm{CoO}_{6}\right]$ octahedra in $\mathrm{SrCo}_{4}(\mathrm{OH})\left(\mathrm{PO}_{4}\right)_{3}$ are distorted, with the $\mathrm{Co}-\mathrm{O}$ distance varying between 2.022 (2) and 2.284 (2) $\AA$. The averaged Co$\mathrm{O}$ distances of $2.130 \AA$ for $\mathrm{Co} 1,2.122 \AA$ for $\mathrm{Co} 2$ and $2.124 \AA$ for $\mathrm{Co} 3$ are in good agreement with those of $\mathrm{Co}_{5}\left(\mathrm{PO}_{4}\right)_{2}(\mathrm{OH})_{4}$ (average $\mathrm{Co}-\mathrm{O}$ distances are $2.107 \AA$ for Co1, $2.144 \AA$ for $\mathrm{Co} 2,2.140 \AA$ for $\mathrm{Co} 3,2.148 \AA$ for $\mathrm{Co} 4$ and $2.150 \AA$ for $\mathrm{Co} 5$; Ruszala et al., 1977). The distorted $\left[\mathrm{Co}_{4} \mathrm{O}_{3} \mathrm{OH}\right]$ tetrahedron shows much shorter $\mathrm{Co}-\mathrm{O}$ distances ranging from 1.942 (2) to 1.995 (2) $\AA$. These distances are comparable with the averaged ${ }^{[4]} \mathrm{Co}-\mathrm{O}$ distances of $1.966,1.955,1.957$ and $1.958 \AA$ observed, respectively, in the phosphates $\mathrm{NaCoPO}_{4}, \mathrm{KCoPO}_{4}$, $\mathrm{NH}_{4} \mathrm{CoPO}_{4}$-Hex and $\mathrm{NH}_{4} \mathrm{CoPO}_{4}-\mathrm{ABW}$ (Feng et al., 1997). The $\mathrm{PO}_{4}$ tetrahedra in the title structure have averaged distances of $1.542 \AA$ for $\mathrm{P} 1,1.539 \AA$ for $\mathrm{P} 2$ and $1.539 \AA$ for $\mathrm{P} 3$, and are compatible with the $\mathrm{P}-\mathrm{O}$ distances in the orthophosphate $\mathrm{SrCo}_{2} \mathrm{Fe}\left(\mathrm{PO}_{4}\right)_{3}$ (Bouraima et al., 2016).

The structure model of $\mathrm{SrCo}_{4}(\mathrm{OH})\left(\mathrm{PO}_{4}\right)_{3}$ is in good agreement with calculations of the bond-valence sums (Brown \& Altermatt, 1985). The obtained values (in valence units) for the cations $\mathrm{Sr}^{\mathrm{II}}, \mathrm{Co}^{\mathrm{II}}$, and $\mathrm{P}^{\mathrm{V}}$ are close to the expected values: Sr1 (1.95), Co1 (1.89), Co2 (1.89), Co3 (1.88), Co4 (1.92), P1 (4.90), P2 (4.95) and P3 (4.95). The bond-valence sums calculated for the oxygen atoms range between 1.82 and 2.29 valence units.

\section{Infrared spectroscopy}

An infrared spectrum of $\mathrm{SrCO}_{4}(\mathrm{OH})\left(\mathrm{PO}_{4}\right)_{3}$ was recorded in order to verify the existence of the hydroxyl and $\mathrm{PO}_{4}$ groups in the title compound (Fig. 6). The FT-IR spectrum shows characteristic vibration bands of isolated $\mathrm{PO}_{4}$ groups. The bands observed at around 420 and $463 \mathrm{~cm}^{-1}$ can be assigned to the $v_{2}$ asymmetric stretching mode while the vibration at $573 \mathrm{~cm}^{-1}$ is attributed to $v_{4}$ asymmetric $\mathrm{O}-\mathrm{P}-\mathrm{O}$ deformation. The weak band observed at $750 \mathrm{~cm}^{-1}$ most likely originates from ${ }^{[4]} \mathrm{Co}-\mathrm{O}$ vibrations, as observed in many other phosphates (Rusakov et al., 2006; Antony et al., 2011; Bushiri et al., 2002; De Pedro et al., 2010). The vibration at $1014 \mathrm{~cm}^{-1}$ corresponds to the $v_{3}$ asymmetric stretching mode of the phosphate tetrahedra. The remaining vibrations centred at 3566,3433 and $1632 \mathrm{~cm}^{-1}$ are commonly assigned to the stretching vibration of the bridging $-\mathrm{OH}$ group, as in $\mathrm{Co}_{2} \mathrm{PO}_{4} \mathrm{OH}$ (Wang et al., 2014), in addition to the $\mathrm{OH}^{-}$ librational mode, which is observed at $637 \mathrm{~cm}^{-1}$. We also note the presence of bands at 1384 and $875 \mathrm{~cm}^{-1}$, indicating C-O bonds (Ribeiro et al., 2006). This observation suggests that the powdered sample contained impurities of a carbonate. The assignments of all vibration bands are summarized in Table 2.
Table 2

Assignments of infrared vibration bands $\left(\mathrm{cm}^{-1}\right)$ for $\operatorname{SrCo}_{4}(\mathrm{OH})\left(\mathrm{PO}_{4}\right)_{3}$.

\begin{tabular}{ll}
\hline Position & Assignment \\
\hline 420 & $\nu_{2}$ asymmetric stretching mode of $\mathrm{P}-\mathrm{O}$ bonds \\
463 & $\nu_{2}$ asymmetric stretching mode of $\mathrm{P}-\mathrm{O}$ bond \\
573 & $v_{4}$ asymmetric deformation of $\mathrm{O}-\mathrm{P}-\mathrm{O}$ \\
637 & $\nu_{\mathrm{L}}$ libration mode of the hydroxyl group \\
750 & ${ }^{[4]} \mathrm{Co}-\mathrm{O}$ stretching mode \\
875 & $\nu_{2}$ vibration of $\mathrm{C}-\mathrm{O}$ bond \\
1014 & $\nu_{3}$ asymmetric stretching mode of $\mathrm{PO}_{4}{ }^{3-}$ \\
1384 & $\nu_{3}$ vibration of $\mathrm{C}-\mathrm{O}$ bond \\
1632 & $\mathrm{O}-\mathrm{H}$ stretching band \\
3433 & $\mathrm{O}-\mathrm{H}$ stretching band \\
3566 & $v_{\mathrm{s}}$ stretching mode of the hydroxyl group \\
\hline
\end{tabular}

\section{Database survey}

A search in the Inorganic Crystal Structure Database (ICSD; Zagorac et al., 2019) revealed no match in the pseudoquaternary system $\mathrm{SrO} / \mathrm{CoO} / \mathrm{P}_{2} \mathrm{O}_{5} / \mathrm{OH}$. However, five compounds were identified in the pseudo-ternary $\mathrm{SrO} / \mathrm{CoO} /$ $\mathrm{P}_{2} \mathrm{O}_{5}$ system, viz. triclinic $\mathrm{SrCo}_{2}\left(\mathrm{PO}_{4}\right)_{2}(P \overline{1}, Z=2$; El Bali et al., 1993), monoclinic $\mathrm{SrCoP}_{2} \mathrm{O}_{7}\left(P 2_{1} / n, Z=4\right.$; Riou \& Raveau 1991), monoclinic $\mathrm{Sr}_{2} \mathrm{Co}\left(\mathrm{PO}_{4}\right)_{2}$ and $\mathrm{SrCo}_{3}\left(\mathrm{P}_{2} \mathrm{O}_{7}\right)_{2}$ (both $P 2_{1} / c$, $Z=6$ and 2, respectively; Belik et al. 2001 and Yang et al., 2008) and hexagonal $\mathrm{Sr}_{5} \mathrm{Co}_{0.18} \mathrm{P}_{3} \mathrm{O}_{12.92}\left(P 6_{3} / m, Z=2\right.$; Kazin et al., 2017).

\section{Synthesis and crystallization}

Single crystals of $\mathrm{SrCo}_{4}(\mathrm{OH})\left(\mathrm{PO}_{4}\right)_{3}$ were obtained serendipitously by attempting to synthesize the borophosphate $\mathrm{SrCo}_{2} \mathrm{BPO}_{7}$ under hydrothermal conditions. The starting materials, $\quad \mathrm{Sr}\left(\mathrm{NO}_{3}\right)_{2} \quad(0.3174 \mathrm{~g}), \quad \mathrm{Co}\left(\mathrm{CH}_{3} \mathrm{COO}\right)_{2} \cdot 4 \mathrm{H}_{2} \mathrm{O}$ $(0.7473 \mathrm{~g}), \mathrm{H}_{3} \mathrm{BO}_{3}(0.0927 \mathrm{~g})$ and $\mathrm{H}_{3} \mathrm{PO}_{4}(12 \mathrm{~N} ; 0.1 \mathrm{ml})$, were mixed in the molar proportions of 1:2:1:1. The hydrothermal reaction was conducted in a $23 \mathrm{ml}$ Teflon-lined autoclave, filled to $50 \%$ with distilled water and heated under autogenous pressure at $473 \mathrm{~K}$ for five days. After the end of the heat treatment, the autoclave was taken out of the oven and allowed to cool to room temperature. The reaction product

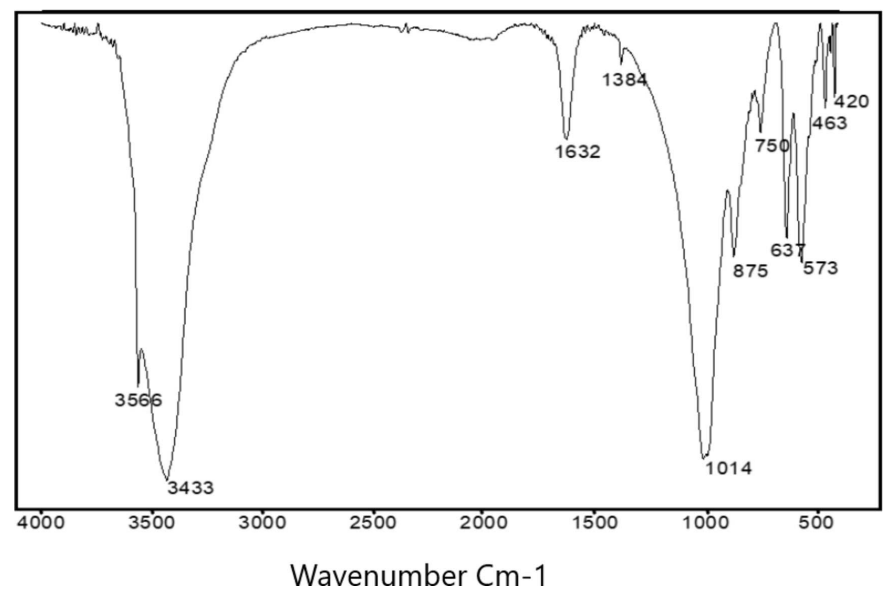

Figure 6

Infrared spectrum of $\mathrm{SrCo}_{4}(\mathrm{OH})\left(\mathrm{PO}_{4}\right)_{3}$. 
Table 3

Experimental details.

\begin{tabular}{|c|c|}
\hline \multicolumn{2}{|l|}{ Crystal data } \\
\hline Chemical formula & $\mathrm{SrCo}_{4}(\mathrm{OH})\left(\mathrm{PO}_{4}\right)_{3}$ \\
\hline$M_{\mathrm{r}}$ & 625.26 \\
\hline Crystal system, space group & Orthorhombic, $P 2_{1} 2_{1} 2_{1}$ \\
\hline Temperature $(\mathrm{K})$ & 296 \\
\hline$a, b, c(\AA)$ & $5.1245(1), 12.0491(2), 15.7118(3)$ \\
\hline$V\left(\AA^{3}\right)$ & $970.13(3)$ \\
\hline$Z$ & 4 \\
\hline Radiation type & Mo $K \alpha$ \\
\hline$\mu\left(\mathrm{mm}^{-1}\right)$ & 12.74 \\
\hline Crystal size $(\mathrm{mm})$ & $0.35 \times 0.26 \times 0.17$ \\
\hline \multicolumn{2}{|l|}{ Data collection } \\
\hline Diffractometer & Bruker X8 APEX \\
\hline Absorption correction & $\begin{array}{l}\text { Multi-scan (SADABS; Krause et } \\
\quad \text { al., 2015) }\end{array}$ \\
\hline$T_{\min }, T_{\max }$ & $0.391,0.748$ \\
\hline $\begin{array}{l}\text { No. of measured, independent and } \\
\text { observed }[I>2 \sigma(I)] \text { reflections }\end{array}$ & $34471,4243,4038$ \\
\hline$R_{\text {int }}$ & 0.045 \\
\hline$(\sin \theta / \lambda)_{\max }\left(\AA^{-1}\right)$ & 0.806 \\
\hline \multicolumn{2}{|l|}{ Refinement } \\
\hline$R\left[F^{2}>2 \sigma\left(F^{2}\right)\right], w R\left(F^{2}\right), S$ & $0.022,0.050,1.07$ \\
\hline No. of reflections & 4243 \\
\hline No. of parameters & 191 \\
\hline H-atom treatment & $\mathrm{H}$-atom parameters constrained \\
\hline$\Delta \rho_{\max }, \Delta \rho_{\min }\left(\mathrm{e} \AA^{-3}\right)$ & $1.60,-0.68$ \\
\hline Absolute structure & $\begin{array}{l}\text { Flack } x \text { determined using } 1625 \\
\text { quotients }\left[\left(I^{+}\right)-\left(I^{-}\right)\right] /\left[\left(I^{+}\right)+\left(I^{-}\right)\right] \\
\text {(Parsons } \text { et al. } 2013) \text {. }\end{array}$ \\
\hline Absolute structure parameter & $0.008(3)$ \\
\hline
\end{tabular}

Computer programs: APEX2 and SAINT (Bruker, 2016), SHELXT2014/5 (Sheldrick, 2015a), SHELXL2018/3 (Sheldrick, 2015b), ORTEP-3 for Windows (Farrugia, 2012), DIAMOND (Brandenburg, 2006) and publCIF (Westrip, 2010).

was collected, filtered, rinsed with distilled water and dried in air. Optical microscopy revealed two types of crystals, viz. dark-purple and dark-red rectangular crystals. X-ray diffraction analysis showed the red crystals to be $\mathrm{Co}_{2}(\mathrm{OH}) \mathrm{PO}_{4}$ (Harrison et al., 1995). The purple parallelepipeds correspond to the title compound.

Infrared spectroscopic measurements were performed on a VERTEX 70 FT-IR spectrometer, using the MRI transmission technique using $\mathrm{KBr}$ pellets. An adequate quantity of the studied phosphate powder, obtained by grinding the $\mathrm{SrCo}_{4}(\mathrm{OH})\left(\mathrm{PO}_{4}\right)_{3}$ crystals, was diluted in $\mathrm{KBr}$ before being pressed into a pellet. The analysis was performed at room temperature, and the spectrum was recorded in the range $4000-400 \mathrm{~cm}^{-1}$.

\section{Refinement}

Crystal data, data collection and structure refinement details are summarized in Table 3. The hydrogen atom of the $\mathrm{OH}$ group was located in a difference-Fourier map and was refined was a fixed $\mathrm{O}-\mathrm{H}$ bond length of $0.82 \AA$ and $U_{\text {iso }}(\mathrm{H})=$ $1.5 U_{\text {eq }}(\mathrm{O})$. The maximum and minimum remaining electron density was located at $0.69 \AA$ from Sr1 and $0.56 \AA$ from $\mathrm{Co} 4$, respectively. The reflection (011) was affected by the beamstop $\left(F_{\mathrm{o}}^{2}=0\right)$ while reflections $(052)$ and $(053)$, having $F_{\mathrm{o}}^{2}>F_{\mathrm{c}}^{2}$, were probably affected by the Renninger effect. All three reflections were omitted from the refinement.

\section{Acknowledgements}

The authors thank the Unit of Support for Technical and Scientific Research (UATRS, CNRST) for the X-ray measurements and Mohammed V University, Rabat, Morocco, for financial support.

\section{References}

Antony, C. J., Aatiq, A., Panicker, C. Y., Bushiri, M. J., Varghese, H. T. \& Manojkumar, T. K. (2011). Spectrochim. Acta Part A, 78, 415419.

Belik, A. A., Lazoryak, B. I., Terekhina, T. P. \& Polyakov, S. N. (2001). Zh. Neorg. Khim. 46, 1453-1459.

Borel, M. M., Goreaud, M., Grandin, A., Labbe', Ph., Leclaire, A. \& Raveau, B. (1991). Eur. J. Solid State Inorg. Chem. 28, 93129.

Bouraima, A., Makani, T., Assani, A., Saadi, M. \& El Ammari, L. (2016). Acta Cryst. E72, 1143-1146.

Brandenburg, K. (2006). DIAMOND. Crystal Impact GbR, Bonn, Germany.

Brown, I. D. \& Altermatt, D. (1985). Acta Cryst. B41, 244-247.

Bruker (2016). APEX3 and SAINT. Bruker AXS Inc., Madison, Wisconsin, USA.

Bushiri, M. J., Jayasree, R. S., Fakhfakh, M. \& Nayar, V. U. (2002). Mater. Chem. Phys. 73, 179-185.

El Bali, B., Boukhari, A., Holt, E. M. \& Aride, J. (1993). J. Crystallogr. Spectrosc. Res. 23, 1001-1004.

Ewald, B., Huang, Y.-X. \& Kniep, R. (2007). Z. Anorg. Allg. Chem. 633, 1517-1540.

Farrugia, L. J. (2012). J. Appl. Cryst. 45, 849-854.

Feng, P., Bu, X., Tolbert, S. H. \& Stucky, G. D. (1997). J. Am. Chem. Soc. 119, 2497-2504.

Goodenough, J. B., Hong, H. Y. P. \& Kafalas, J. A. (1976). Mater. Res. Bull. 11, 203-220.

Hadouchi, M., Assani, A., Saadi, M., Lahmar, A., El Marssi, M. \& El Ammari, L. (2019). Acta Cryst. C75, 777-782.

Harrison, W. T. A., Vaughey, J. T., Dussack, L. L., Jacobson, A. J., Martin, T. E. \& Stucky, G. D. (1995). J. Solid State Chem. 114, 151158.

Kazin, P. E., Zykin, M. A., Schnelle, W., Zubavichus, Y. V., Babeshkin, K. A., Tafeenko, V. A., Felser, C. \& Jansen, M. (2017). Inorg. Chem. 56, 1232-1240.

Khmiyas, J., Benhsina, E., Ouaatta, S., Assani, A., Saadi, M. \& El Ammari, L. (2020). Acta Cryst. E76, 186-191.

Kniep, R., Engelhardt, H. \& Hauf, C. (1998). Chem. Mater. 10, 2930 2934.

Krause, L., Herbst-Irmer, R., Sheldrick, G. M. \& Stalke, D. (2015). J. Appl. Cryst. 48, 3-10.

La Parola, V., , Liveri, V. T., Todaro, L., Lombardo, D., Bauer, E. M., Dell'Era, A., Longo, A., Caschera, D., de Caro, T., Toro, R. G. \& Calandra, P. (2018). Mater. Lett. 220, 58-61.

Lin, J.-R., Huang, Y.-X., Wu, Y.-H. \& Zhou, Y. (2008). Acta Cryst. E64, i39-i40.

Menezes, P. W., Hoffmann, S., Prots, Y. \& Kniep, R. (2008). Z. Kristallogr. 223, 333-334.

Ouaatta, S., Assani, A., Saadi, M. \& El Ammari, L. (2019). Acta Cryst. E75, 402-404.

Parsons, S., Flack, H. D. \& Wagner, T. (2013). Acta Cryst. B69, 249 259.

Pedro, I. de, Rojo, J. M., Rodríguez Fernández, J., Lezama, L. \& Rojo, T. (2010). Eur. J. Inorg. Chem. pp. 2514-2522.

Rghioui, L., El Ammari, L., Assani, A. \& Saadi, M. (2019). Acta Cryst. E75, 1041-1045.

Ribeiro, C. C., Gibson, I. \& Barbosa, M. A. (2006). Biomaterials, 27, 1749-1761. 
Riou, D. \& Raveau, B. (1991). Acta Cryst. C47, 1708-1709.

Rusakov, D. A., Filaretov, A. A., Bubentsova, M. N., Danilov, V. P. \& Komissarova, L. N. (2006). Russ. J. Inorg. Chem. 51, 852-861.

Ruszala, F. A., Anderson, J. B. \& Kostiner, E. (1977). Inorg. Chem. 16, 2417-2422.

Sheldrick, G. M. (2015a). Acta Cryst. A71, 3-8.

Sheldrick, G. M. (2015b). Acta Cryst. C71, 3-8.

Wang, G., Valldor, M., Spielberg, E. T. \& Mudring, A. V. (2014). Inorg. Chem. 53, 3072-3077.
Westrip, S. P. (2010). J. Appl. Cryst. 43, 920-925.

Yang, T., Zhang, Y., Yang, S., Li, G., Xiong, M., Liao, F. \& Lin, J. (2008). Inorg. Chem. 47, 2562-2568.

Zagorac, D., Müller, H., Ruehl, S., Zagorac, J. \& Rehme, S. (2019). J. Appl. Cryst. 52, 918-925.

Zouihri, H., Saadi, M., Jaber, B. \& El Ammari, L. (2011a). Acta Cryst. E67, i44.

Zouihri, H., Saadi, M., Jaber, B. \& El Ammari, L. (2011b). Acta Cryst. E67, i39. 


\section{supporting information}

Acta Cryst. (2020). E76, 1022-1026 [https://doi.org/10.1107/S2056989020007331]

\section{Crystal structure of $\mathrm{SrCO}_{4}(\mathrm{OH})\left(\mathrm{PO}_{4}\right)_{3}$, a new hydroxyphosphate}

\section{Fatima-Zahra Cherif, Mhamed Taibi, Ali Boukhari, Jilali Aride, Abderrazzak Assani, Mohamed Saadi and Lahcen El Ammari}

\section{Computing details}

Data collection: APEX2 (Bruker, 2016); cell refinement: SAINT (Bruker, 2016); data reduction: SAINT (Bruker, 2016); program(s) used to solve structure: SHELXT2014/5 (Sheldrick, 2015a); program(s) used to refine structure: SHELXL2018/3 (Sheldrick, 2015b); molecular graphics: ORTEP-3 for Windows (Farrugia, 2012), DIAMOND (Brandenburg, 2006); software used to prepare material for publication: publCIF (Westrip, 2010).

Strontium tetracobalt tris(orthophosphate) hydroxide

Crystal data

$\mathrm{SrCO}_{4}(\mathrm{OH})\left(\mathrm{PO}_{4}\right)_{3}$

$M_{r}=625.26$

Orthorhombic, $P 2{ }_{1} 22_{1}$

$a=5.1245(1) \AA$

$b=12.0491(2) \AA$

$c=15.7118(3) \AA$

$V=970.13(3) \AA^{3}$

$Z=4$

$F(000)=1184$

\section{Data collection}

Bruker X8 APEX Diffractometer

Radiation source: fine-focus sealed tube

Graphite monochromator

$\varphi$ and $\omega$ scans

Absorption correction: multi-scan

(SADABS; Krause et al., 2015)

$T_{\text {min }}=0.391, T_{\max }=0.748$

34471 measured reflections

Refinement

Refinement on $F^{2}$

Least-squares matrix: full

$R\left[F^{2}>2 \sigma\left(F^{2}\right)\right]=0.022$

$w R\left(F^{2}\right)=0.050$

$S=1.07$

4243 reflections

191 parameters

0 restraints

Hydrogen site location: difference Fourier map

$\mathrm{H}$-atom parameters constrained
$D_{\mathrm{x}}=4.281 \mathrm{Mg} \mathrm{m}^{-3}$

Mo $K \alpha$ radiation, $\lambda=0.71073 \AA$

Cell parameters from 4243 reflections

$\theta=2.6-35.0^{\circ}$

$\mu=12.74 \mathrm{~mm}^{-1}$

$T=296 \mathrm{~K}$

Parallelepiped, violet

$0.35 \times 0.26 \times 0.17 \mathrm{~mm}$

4243 independent reflections

4038 reflections with $I>2 \sigma(I)$

$R_{\text {int }}=0.045$

$\theta_{\max }=35.0^{\circ}, \theta_{\min }=2.6^{\circ}$

$h=-8 \rightarrow 8$

$k=-19 \rightarrow 18$

$l=-25 \rightarrow 25$

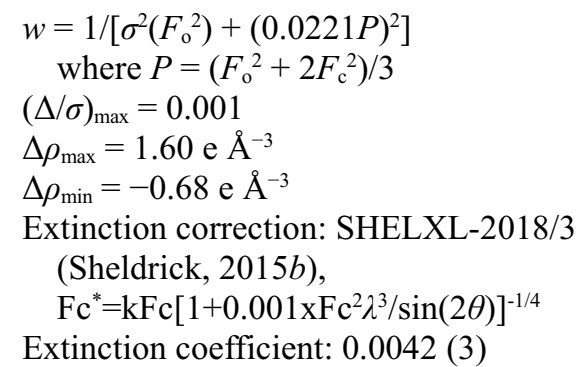


Absolute structure: Flack $x$ determined using

1625 quotients $\left[\left(I^{+}\right)-\left(I^{-}\right)\right] /\left[\left(I^{+}\right)+\left(I^{-}\right)\right]$(Parsons et al., 2013).

Absolute structure parameter: 0.008 (3)

\section{Special details}

Geometry. All esds (except the esd in the dihedral angle between two 1.s. planes) are estimated using the full covariance matrix. The cell esds are taken into account individually in the estimation of esds in distances, angles and torsion angles; correlations between esds in cell parameters are only used when they are defined by crystal symmetry. An approximate (isotropic) treatment of cell esds is used for estimating esds involving l.s. planes.

Fractional atomic coordinates and isotropic or equivalent isotropic displacement parameters $\left(\AA^{2}\right)$

\begin{tabular}{lllll}
\hline & $x$ & $y$ & $z$ & $U_{\text {iso }} * / U_{\text {eq }}$ \\
\hline Sr1 & $0.02677(6)$ & $0.72309(2)$ & $0.67782(2)$ & $0.00840(6)$ \\
Co1 & $0.51704(8)$ & $0.48429(3)$ & $0.35972(3)$ & $0.00572(8)$ \\
Co2 & $0.53401(9)$ & $0.58186(4)$ & $0.54997(3)$ & $0.00613(8)$ \\
Co3 & $0.47299(8)$ & $0.46200(3)$ & $0.74367(3)$ & $0.00580(8)$ \\
Co4 & $0.28203(8)$ & $0.32358(4)$ & $0.51444(3)$ & $0.00794(9)$ \\
P1 & $0.51747(15)$ & $0.85171(6)$ & $0.57349(5)$ & $0.00425(12)$ \\
P2 & $0.01127(15)$ & $0.45034(6)$ & $0.65719(5)$ & $0.00471(13)$ \\
P3 & $-0.00586(15)$ & $0.30856(6)$ & $0.33173(5)$ & $0.00413(12)$ \\
O1 & $0.4003(4)$ & $0.8648(2)$ & $0.48533(15)$ & $0.0092(4)$ \\
O2 & $0.8108(4)$ & $0.8848(2)$ & $0.57323(15)$ & $0.0074(4)$ \\
O3 & $0.3638(4)$ & $0.9243(2)$ & $0.63798(15)$ & $0.0074(4)$ \\
O4 & $0.4924(5)$ & $0.73162(18)$ & $0.60637(15)$ & $0.0095(4)$ \\
O5 & $0.2427(4)$ & $0.5287(2)$ & $0.63664(15)$ & $0.0078(4)$ \\
O6 & $-0.2433(4)$ & $0.5173(2)$ & $0.66027(15)$ & $0.0075(4)$ \\
O7 & $0.0710(4)$ & $0.40910(19)$ & $0.74879(15)$ & $0.0068(4)$ \\
O8 & $-0.0011(5)$ & $0.3572(2)$ & $0.59242(14)$ & $0.0108(4)$ \\
O9 & $0.1926(4)$ & $0.40027(19)$ & $0.30856(15)$ & $0.0073(4)$ \\
O10 & $0.0074(4)$ & $0.29074(18)$ & $0.43053(14)$ & $0.0080(4)$ \\
O11 & $-0.2813(4)$ & $0.34923(19)$ & $0.30965(15)$ & $0.0075(4)$ \\
O12 & $0.0671(4)$ & $0.20305(19)$ & $0.28586(16)$ & $0.0097(4)$ \\
O13 & $0.5519(4)$ & $0.43152(18)$ & $0.48508(15)$ & $0.0073(4)$ \\
H13 & 0.691669 & 0.405651 & 0.500919 & $0.011 *$ \\
& & & & \\
\hline & & & & \\
\hline
\end{tabular}

Atomic displacement parameters $\left(\AA^{2}\right)$

\begin{tabular}{lllllll}
\hline & $U^{11}$ & $U^{22}$ & $U^{33}$ & $U^{12}$ & $U^{13}$ & $U^{23}$ \\
\hline Sr1 & $0.01002(12)$ & $0.00611(12)$ & $0.00908(12)$ & $0.00095(10)$ & $0.00229(10)$ & $0.00061(10)$ \\
Co1 & $0.00609(16)$ & $0.00557(17)$ & $0.00550(17)$ & $-0.00054(14)$ & $-0.00005(14)$ & $-0.00035(13)$ \\
Co2 & $0.00739(17)$ & $0.00508(17)$ & $0.00593(17)$ & $-0.00087(15)$ & $0.00149(14)$ & $-0.00104(13)$ \\
Co3 & $0.00560(16)$ & $0.00635(17)$ & $0.00544(16)$ & $0.00038(14)$ & $0.00019(14)$ & $0.00037(13)$ \\
Co4 & $0.00687(17)$ & $0.0095(2)$ & $0.00748(18)$ & $-0.00059(14)$ & $-0.00076(14)$ & $0.00135(16)$ \\
P1 & $0.0047(3)$ & $0.0041(3)$ & $0.0039(3)$ & $-0.0005(3)$ & $0.0001(2)$ & $0.0001(2)$ \\
P2 & $0.0044(3)$ & $0.0055(3)$ & $0.0042(3)$ & $0.0001(3)$ & $-0.0001(2)$ & $0.0002(2)$ \\
P3 & $0.0051(3)$ & $0.0035(3)$ & $0.0038(3)$ & $-0.0002(2)$ & $-0.0002(2)$ & $0.0000(2)$ \\
O1 & $0.0093(9)$ & $0.0138(11)$ & $0.0045(9)$ & $0.0012(8)$ & $-0.0026(8)$ & $-0.0010(9)$
\end{tabular}




\begin{tabular}{lllllll}
\hline & & & & \\
O2 & $0.0044(9)$ & $0.0098(10)$ & $0.0079(10)$ & $-0.0026(8)$ & $0.0002(7)$ & $-0.0008(8)$ \\
O3 & $0.0069(9)$ & $0.0083(10)$ & $0.0071(10)$ & $0.0033(8)$ & $-0.0001(7)$ & $-0.0022(9)$ \\
O4 & $0.0140(10)$ & $0.0036(9)$ & $0.0109(9)$ & $-0.0001(9)$ & $0.0049(8)$ & $0.0008(7)$ \\
O5 & $0.0058(9)$ & $0.0099(10)$ & $0.0077(10)$ & $-0.0020(8)$ & $0.0004(7)$ & $0.0028(9)$ \\
O6 & $0.0061(9)$ & $0.0099(11)$ & $0.0065(10)$ & $0.0027(8)$ & $-0.0002(7)$ & $0.0016(8)$ \\
O7 & $0.0069(9)$ & $0.0073(10)$ & $0.0062(9)$ & $-0.0016(7)$ & $-0.0012(7)$ & $0.0015(8)$ \\
O8 & $0.0115(10)$ & $0.0114(10)$ & $0.0094(10)$ & $-0.0022(9)$ & $0.0030(9)$ & $-0.0059(8)$ \\
O9 & $0.0077(9)$ & $0.0055(9)$ & $0.0088(10)$ & $-0.0020(7)$ & $0.0004(7)$ & $0.0022(8)$ \\
O10 & $0.0095(10)$ & $0.0104(10)$ & $0.0039(9)$ & $-0.0020(8)$ & $0.0000(8)$ & $0.0008(7)$ \\
O11 & $0.0062(9)$ & $0.0081(10)$ & $0.0082(10)$ & $0.0008(8)$ & $-0.0016(7)$ & $-0.0017(8)$ \\
O12 & $0.0120(10)$ & $0.0060(10)$ & $0.0111(10)$ & $0.0014(8)$ & $-0.0002(8)$ & $-0.0035(8)$ \\
O13 & $0.0068(9)$ & $0.0058(9)$ & $0.0092(9)$ & $0.0006(7)$ & $-0.0002(8)$ & $0.0003(8)$ \\
\end{tabular}

Geometric parameters $\left(\AA,{ }^{\circ}\right)$

\begin{tabular}{|c|c|c|c|}
\hline $\mathrm{Sr} 1-\mathrm{O}^{\mathrm{i}}$ & $2.570(2)$ & $\mathrm{Co} 3-\mathrm{O}^{\mathrm{vi}}$ & $2.067(2)$ \\
\hline $\mathrm{Sr} 1-\mathrm{O} 11^{\mathrm{ii}}$ & $2.575(2)$ & $\mathrm{Co} 3-\mathrm{O} 3^{\mathrm{ix}}$ & $2.089(2)$ \\
\hline $\mathrm{Sr} 1-\mathrm{O} 4$ & $2.639(2)$ & $\mathrm{Co} 3-\mathrm{O} 12^{\mathrm{x}}$ & $2.098(2)$ \\
\hline $\mathrm{Sr} 1-\mathrm{O} 5$ & $2.669(2)$ & $\mathrm{Co} 3-\mathrm{O}^{\mathrm{iv}}$ & $2.125(2)$ \\
\hline $\mathrm{Sr} 1-\mathrm{O} 2^{\mathrm{iii}}$ & $2.779(2)$ & $\mathrm{Co} 3-\mathrm{O} 7$ & $2.158(2)$ \\
\hline $\mathrm{Sr} 1-\mathrm{O} 12^{\mathrm{iv}}$ & $2.829(2)$ & $\mathrm{Co} 3-\mathrm{O} 5$ & $2.206(2)$ \\
\hline $\mathrm{Sr} 1-\mathrm{O}^{\mathrm{v}}$ & $2.848(2)$ & $\mathrm{Co} 4-\mathrm{O} 8$ & $1.942(2)$ \\
\hline $\mathrm{Sr} 1-\mathrm{O} 6$ & $2.853(2)$ & $\mathrm{Co} 4-\mathrm{O} 13$ & $1.954(2)$ \\
\hline $\mathrm{Sr} 1-\mathrm{O} 9^{\mathrm{iv}}$ & $2.915(2)$ & $\mathrm{Co} 4-\mathrm{O} 10$ & $1.969(2)$ \\
\hline $\mathrm{Sr} 1-\mathrm{O} 4^{\mathrm{iii}}$ & $2.961(2)$ & $\mathrm{Co} 4-\mathrm{O} 10^{\mathrm{x}}$ & $1.995(2)$ \\
\hline $\mathrm{Sr} 1-\mathrm{O} 3$ & $3.041(2)$ & $\mathrm{P} 1-\mathrm{O} 1$ & $1.518(2)$ \\
\hline $\mathrm{Co} 1-\mathrm{O} 13$ & $2.077(2)$ & $\mathrm{P} 1-\mathrm{O} 4$ & $1.542(2)$ \\
\hline $\mathrm{Co} 1-\mathrm{O} 11^{\mathrm{vi}}$ & $2.082(2)$ & $\mathrm{P} 1-\mathrm{O} 3$ & $1.553(2)$ \\
\hline $\mathrm{Co} 1-\mathrm{O}^{\mathrm{vii}}$ & $2.091(2)$ & $\mathrm{P} 1-\mathrm{O} 2$ & $1.555(2)$ \\
\hline $\mathrm{Co} 1-\mathrm{O} 9$ & $2.106(2)$ & $\mathrm{P} 2-\mathrm{O} 8$ & $1.517(2)$ \\
\hline $\mathrm{Co} 1-\mathrm{O} 2^{\mathrm{v}}$ & $2.171(2)$ & $\mathrm{P} 2-\mathrm{O} 6$ & $1.534(2)$ \\
\hline $\mathrm{Co} 1-\mathrm{O} 7^{\text {viii }}$ & $2.212(2)$ & $\mathrm{P} 2-\mathrm{O} 5$ & $1.550(2)$ \\
\hline $\mathrm{Co} 2-\mathrm{O} 4$ & $2.022(2)$ & $\mathrm{P} 2-\mathrm{O} 7$ & $1.553(2)$ \\
\hline $\mathrm{Co} 2-\mathrm{O} 1^{\mathrm{vii}}$ & $2.060(2)$ & $\mathrm{P} 3-\mathrm{O} 12$ & $1.508(2)$ \\
\hline $\mathrm{Co} 2-\mathrm{O} 13$ & $2.081(2)$ & P3-O11 & $1.534(2)$ \\
\hline $\mathrm{Co} 2-\mathrm{O} 5$ & $2.120(2)$ & $\mathrm{P} 3-\mathrm{O} 9$ & $1.545(2)$ \\
\hline $\mathrm{Co} 2-\mathrm{O}^{\mathrm{vi}}$ & $2.216(2)$ & $\mathrm{P} 3-\mathrm{O} 10$ & $1.569(2)$ \\
\hline $\mathrm{Co} 2-\mathrm{O} 2^{v}$ & $2.284(2)$ & $\mathrm{O} 13-\mathrm{H} 13$ & 0.8200 \\
\hline $\mathrm{O} 7^{\mathrm{i}}-\mathrm{Sr} 1-\mathrm{O} 11^{\mathrm{ii}}$ & $80.73(7)$ & $\mathrm{O} 9-\mathrm{Co} 1-\mathrm{O} 2^{\mathrm{v}}$ & $98.63(9)$ \\
\hline $\mathrm{O} 7^{\mathrm{i}}-\mathrm{Sr} 1-\mathrm{O} 4$ & $109.46(7)$ & $\mathrm{O} 13-\mathrm{Co} 1-\mathrm{O} 7^{\text {viii }}$ & $160.47(9)$ \\
\hline $\mathrm{O} 11^{\mathrm{ii}}-\mathrm{Sr} 1-\mathrm{O} 4$ & $142.85(7)$ & $\mathrm{O} 11^{\mathrm{vi}}-\mathrm{Co} 1-\mathrm{O} 7^{\mathrm{viii}}$ & $104.92(9)$ \\
\hline $\mathrm{O} 7-\mathrm{i} r 1-\mathrm{O} 5$ & $162.67(7)$ & $\mathrm{O} 3^{\text {vii }}-\mathrm{Co} 1-\mathrm{O} 7^{\text {viii }}$ & $83.16(8)$ \\
\hline $\mathrm{O} 11^{\mathrm{ii}}-\mathrm{Sr} 1-\mathrm{O} 5$ & $95.77(7)$ & $\mathrm{O} 9-\mathrm{Co} 1-\mathrm{O} 7^{\text {viii }}$ & $79.48(8)$ \\
\hline $\mathrm{O} 4-\mathrm{Sr} 1-\mathrm{O} 5$ & $63.66(7)$ & $\mathrm{O} 2^{\mathrm{v}}-\mathrm{Co} 1-\mathrm{O} 7^{\mathrm{viii}}$ & $82.02(9)$ \\
\hline $\mathrm{O} 7^{\mathrm{i}}-\mathrm{Sr} 1-\mathrm{O} 2^{\mathrm{iii}}$ & $64.92(7)$ & $\mathrm{O} 4-\mathrm{Co} 2-\mathrm{O}^{\mathrm{vii}}$ & $86.30(10)$ \\
\hline $\mathrm{O} 11^{\mathrm{ii}}-\mathrm{Sr} 1-\mathrm{O} 2^{\mathrm{iii}}$ & $121.22(7)$ & $\mathrm{O} 4-\mathrm{Co} 2-\mathrm{O} 13$ & $175.22(10)$ \\
\hline $\mathrm{O} 4-\mathrm{Sr} 1-\mathrm{O} 2^{\mathrm{iii}}$ & $94.66(7)$ & $\mathrm{O} 1^{\mathrm{vi}}-\mathrm{Co} 2-\mathrm{O} 13$ & $95.72(9)$ \\
\hline
\end{tabular}




$$
\begin{aligned}
& \mathrm{O} 5-\mathrm{Sr} 1-\mathrm{O} 2^{\mathrm{iii}} \\
& \mathrm{O} 7{ }^{\mathrm{i}}-\mathrm{Sr} 1-\mathrm{O} 12^{\mathrm{iv}} \\
& \mathrm{O} 11^{\mathrm{ii}}-\mathrm{Sr} 1-\mathrm{O} 12^{\mathrm{iv}} \\
& \mathrm{O} 4-\mathrm{Sr} 1-\mathrm{O} 12^{\mathrm{iv}} \\
& \mathrm{O} 5-\mathrm{Sr} 1-\mathrm{O} 12^{\mathrm{iv}} \\
& \mathrm{O} 2^{\mathrm{iii}}-\mathrm{Sr} 1-\mathrm{O} 12^{\mathrm{iv}} \\
& \mathrm{O} 7^{\mathrm{i}}-\mathrm{Sr} 1-\mathrm{O}^{\mathrm{v}} \\
& \mathrm{O} 11^{\mathrm{ii}}-\mathrm{Sr} 1-\mathrm{O}^{\mathrm{v}} \\
& \mathrm{O} 4-\mathrm{Sr} 1-\mathrm{O}^{\mathrm{v}} \\
& \mathrm{O} 5-\mathrm{Sr} 1-\mathrm{O}^{\mathrm{v}}
\end{aligned}
$$

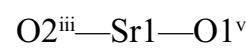

$$
\begin{aligned}
& \mathrm{O} 12^{\mathrm{iv}}-\mathrm{Sr} 1-\mathrm{O}^{\mathrm{v}} \\
& \text { O7 }-\mathrm{Sr} 1-\mathrm{O} 6 \\
& \mathrm{O} 11^{\mathrm{ii}}-\mathrm{Sr} 1-\mathrm{O} 6 \\
& \text { O4- } \mathrm{Sr} 1-\mathrm{O} 6 \\
& \text { O5- } \mathrm{Sr} 1-\mathrm{O} 6 \\
& \mathrm{O} 2{ }^{\mathrm{iii}-\mathrm{Sr} 1-\mathrm{O} 6} \\
& \mathrm{O} 12^{\mathrm{iv}} \text { - } \mathrm{Sr} 1-\mathrm{O} 6 \\
& \text { O1 }-\mathrm{Sr} 1-\mathrm{O} 6 \\
& \mathrm{O} 7^{\mathrm{i}}-\mathrm{Sr} 1-\mathrm{O}^{\mathrm{iv}} \\
& \text { O11 } 1 \text { ii } \mathrm{Sr} 1-\mathrm{O}^{\text {iv }} \\
& \mathrm{O} 4-\mathrm{Sr} 1-\mathrm{O}^{\text {iv }} \\
& \mathrm{O} 5-\mathrm{Sr} 1-\mathrm{O}^{\text {iv }} \\
& \mathrm{O} 2^{\mathrm{iii}}-\mathrm{Sr} 1-\mathrm{O}^{\mathrm{iv}} \\
& \text { O12 }{ }^{\text {iv }}-\mathrm{Sr} 1-\mathrm{O}^{\text {iv }} \\
& \mathrm{O} 1^{\mathrm{v}}-\mathrm{Sr} 1-\mathrm{O}^{\mathrm{iv}} \\
& \text { O6- } \mathrm{Sr} 1-\mathrm{O}^{\mathrm{iv}} \\
& \mathrm{O} 7^{\mathrm{i}}-\mathrm{Sr} 1-\mathrm{O}^{\mathrm{iii}} \\
& \mathrm{O} 11^{\mathrm{ii}}-\mathrm{Sr} 1-\mathrm{O} 4^{\mathrm{iii}} \\
& \mathrm{O} 4-\mathrm{Sr} 1-\mathrm{O} 4^{\mathrm{iii}} \\
& \mathrm{O} 5-\mathrm{Sr} 1-\mathrm{O} 4^{\mathrm{iii}} \\
& \mathrm{O} 2^{i i i}-\mathrm{Sr} 1-\mathrm{O} 4^{\mathrm{iii}} \\
& \mathrm{O} 12^{\mathrm{iv}}-\mathrm{Sr} 1-\mathrm{O} 4^{\mathrm{iii}} \\
& \mathrm{O} 1^{\mathrm{v}}-\mathrm{Sr} 1-\mathrm{O} 4^{\mathrm{iii}} \\
& \text { O6- } \mathrm{Sr} 1-\mathrm{O} 4^{\mathrm{iii}} \\
& \mathrm{O}^{\mathrm{iv}}-\mathrm{Sr} 1-\mathrm{O} 4^{\mathrm{iii}} \\
& \mathrm{O}^{\mathrm{i}}-\mathrm{Sr} 1-\mathrm{O} 3 \\
& \mathrm{O} 11^{\mathrm{ii}}-\mathrm{Sr} 1-\mathrm{O} 3 \\
& \mathrm{O} 4-\mathrm{Sr} 1-\mathrm{O} 3 \\
& \mathrm{O} 5-\mathrm{Sr} 1-\mathrm{O} 3 \\
& \mathrm{O} 2{ }^{\mathrm{iii}-\mathrm{Sr} 1-\mathrm{O} 3} \\
& \mathrm{O} 12^{\mathrm{iv}}-\mathrm{Sr} 1-\mathrm{O} 3 \\
& \mathrm{O} 1 \text { - }-\mathrm{Sr} 1-\mathrm{O} 3 \\
& \text { O6- }-\mathrm{Sr} 1-\mathrm{O} 3 \\
& \mathrm{O}^{\mathrm{iv}}-\mathrm{Sr} 1-\mathrm{O} 3
\end{aligned}
$$

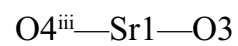

$$
\begin{aligned}
& \text { O13-Co1-O11 }{ }^{\mathrm{vi}} \\
& \mathrm{O} 13-\mathrm{Co} 1-\mathrm{O}^{\text {vii }}
\end{aligned}
$$

$129.56(7)$

$66.42(7)$

$89.04(7)$

$65.03(7)$

96.69 (7)

$115.29(7)$

133.13 (7)

119.13 (7)

80.64 (7)

$63.27(7)$

$68.76(7)$

$145.52(7)$

$134.98(7)$

$63.04(7)$

115.55 (7)

$54.21(6)$

111.05 (7)

133.49 (7)

$58.64(7)$

102.99 (7)

$60.11(7)$

$82.74(7)$

$61.25(7)$

$166.11(7)$

$51.30(6)$

123.85 (7)

$82.19(6)$

$87.68(6)$

82.23 (7)

132.33 (9)

108.78 (7)

$51.92(6)$

$153.73(7)$

$57.40(6)$

$62.94(6)$

$137.82(6)$

$60.53(7)$

135.45 (7)

$50.80(6)$

114.45 (6)

$62.97(6)$

$56.98(7)$

$103.90(6)$

$161.48(6)$

$105.74(6)$

114.80 (6)

$94.38(9)$

$94.12(9)$
$\mathrm{O} 4-\mathrm{Co} 2-\mathrm{O} 5$

$\mathrm{O} 1$ vii- $\mathrm{Co} 2-\mathrm{O} 5$

$\mathrm{O} 13-\mathrm{Co} 2-\mathrm{O} 5$

$\mathrm{O} 4-\mathrm{Co} 2-\mathrm{O}^{\mathrm{vi}}$

$\mathrm{O} 1^{\mathrm{vii}}-\mathrm{Co} 2-\mathrm{O}^{\mathrm{vi}}$

$\mathrm{O} 13-\mathrm{Co} 2-\mathrm{O}^{\mathrm{vi}}$

$\mathrm{O} 5-\mathrm{Co} 2-\mathrm{O}^{\mathrm{vi}}$

$\mathrm{O} 4-\mathrm{Co} 2-\mathrm{O}^{\mathrm{v}}$

$\mathrm{O} 1^{\mathrm{vii}}-\mathrm{Co} 2-\mathrm{O} 2^{\mathrm{v}}$

$\mathrm{O} 13-\mathrm{Co} 2-\mathrm{O}^{\mathrm{v}}$

$\mathrm{O} 5-\mathrm{Co} 2-\mathrm{O}^{\mathrm{v}}$

$\mathrm{O}^{\mathrm{vi}}-\mathrm{Co} 2-\mathrm{O}^{\mathrm{v}}$

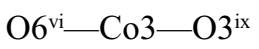

$\mathrm{O} 6^{\mathrm{vi}}-\mathrm{Co} 3-\mathrm{O} 12^{\mathrm{x}}$

$\mathrm{O} 3^{\mathrm{ix}}-\mathrm{Co} 3-\mathrm{O} 12^{\mathrm{x}}$

$\mathrm{O}^{\mathrm{vi}}-\mathrm{Co} 3-\mathrm{O}^{\mathrm{iv}}$

$\mathrm{O}^{\mathrm{ix}}-\mathrm{Co} 3-\mathrm{O}^{\mathrm{iv}}$

$\mathrm{O} 12^{\mathrm{x}}-\mathrm{Co} 3-\mathrm{O}^{\mathrm{iv}}$

$\mathrm{O}^{\mathrm{vi}}-\mathrm{Co} 3-\mathrm{O} 7$

$\mathrm{O} 3^{\mathrm{ix}}-\mathrm{Co} 3-\mathrm{O} 7$

$\mathrm{O} 12^{\mathrm{x}}-\mathrm{Co} 3-\mathrm{O} 7$

$\mathrm{O} 9^{\mathrm{iv}}-\mathrm{Co} 3-\mathrm{O} 7$

$\mathrm{O}^{\mathrm{vi}}-\mathrm{Co} 3-\mathrm{O} 5$

$\mathrm{O} 3^{\mathrm{ix}}-\mathrm{Co} 3-\mathrm{O} 5$

$\mathrm{O} 12^{\mathrm{x}}-\mathrm{Co} 3-\mathrm{O} 5$

$\mathrm{O}^{\mathrm{iv}}-\mathrm{Co} 3-\mathrm{O} 5$

$\mathrm{O} 7-\mathrm{Co} 3-\mathrm{O} 5$

$\mathrm{O} 8-\mathrm{Co} 4-\mathrm{O} 13$

$\mathrm{O} 8-\mathrm{Co} 4-\mathrm{O} 10$

$\mathrm{O} 13-\mathrm{Co} 4-\mathrm{O} 10$

$\mathrm{O} 8-\mathrm{Co} 4-\mathrm{O} 10^{\mathrm{x}}$

O13-Co4-O10

$\mathrm{O} 10-\mathrm{Co} 4-\mathrm{O} 10^{\mathrm{x}}$

$\mathrm{O} 1-\mathrm{P} 1-\mathrm{O} 4$

$\mathrm{O} 1-\mathrm{P} 1-\mathrm{O} 3$

$\mathrm{O} 4-\mathrm{P} 1-\mathrm{O} 3$

$\mathrm{O} 1-\mathrm{P} 1-\mathrm{O} 2$

$\mathrm{O} 4-\mathrm{P} 1-\mathrm{O} 2$

$\mathrm{O} 3-\mathrm{P} 1-\mathrm{O} 2$

$\mathrm{O} 8-\mathrm{P} 2-\mathrm{O} 6$

$\mathrm{O} 8-\mathrm{P} 2-\mathrm{O} 5$

$\mathrm{O} 6-\mathrm{P} 2-\mathrm{O} 5$

$\mathrm{O} 8-\mathrm{P} 2-\mathrm{O} 7$

O6-P2-O7

$\mathrm{O} 5-\mathrm{P} 2-\mathrm{O} 7$

$\mathrm{O} 12-\mathrm{P} 3-\mathrm{O} 11$

O12-P3-O9

O11-P3-O9
85.04 (9)

155.36 (9)

$94.75(9)$

91.44 (10)

81.43 (9)

$93.14(9)$

75.77 (9)

$99.31(9)$

$99.98(9)$

76.10 (9)

104.17 (9)

$169.22(9)$

110.65 (9)

$90.20(10)$

$84.17(10)$

109.48 (9)

84.42 (9)

$159.76(10)$

142.20 (9)

106.54 (9)

87.01 (9)

80.30 (9)

77.04 (9)

166.41 (9)

$107.46(10)$

$82.38(9)$

$68.00(8)$

$122.63(10)$

86.00 (10)

118.80 (9)

$107.64(10)$

$98.76(9)$

$124.43(5)$

$111.72(14)$

$109.66(13)$

$105.51(13)$

110.70 (14)

108.80 (14)

$110.33(13)$

112.00 (14)

110.08 (14)

109.67 (13)

$113.16(14)$

107.85 (13)

$103.72(12)$

112.92 (13)

109.08 (14)

$108.90(13)$ 


$\begin{array}{llll}\mathrm{O}_{1} 1^{\mathrm{vi}}-\mathrm{Co} 1-\mathrm{O} 3^{\mathrm{vii}} & 89.80(9) & \mathrm{O} 12-\mathrm{P} 3-\mathrm{O} 10 & 110.29(14) \\ \mathrm{O} 13-\mathrm{Co} 1-\mathrm{O} 9 & 106.41(9) & \mathrm{O} 11-\mathrm{P} 3-\mathrm{O} 10 & 107.90(13) \\ \mathrm{O} 11^{\mathrm{vi}}-\mathrm{Co} 1-\mathrm{O} 9 & 82.65(9) & \mathrm{O} 9-\mathrm{P} 3-\mathrm{O} 10 & 107.61(13) \\ \mathrm{O} 3^{\mathrm{vii}}-\mathrm{Co} 1-\mathrm{O} 9 & 158.54(9) & \mathrm{Co} 4-\mathrm{O} 13-\mathrm{H} 13 & 107.1 \\ \mathrm{O} 13-\mathrm{Co} 1-\mathrm{O} 2^{\mathrm{v}} & 78.70(9) & \mathrm{Co} 1-\mathrm{O} 13-\mathrm{H} 13 & 118.6 \\ \mathrm{O} 11^{\mathrm{vi}}-\mathrm{Co} 1-\mathrm{O} 2^{\mathrm{v}} & 173.06(9) & \mathrm{Co} 2-\mathrm{O} 13-\mathrm{H} 13 & 102.7 \\ \mathrm{O}^{\mathrm{vii}}-\mathrm{Co} 1-\mathrm{O} 2^{\mathrm{v}} & 91.29(9) & & \end{array}$

Symmetry codes: (i) $-x, y+1 / 2,-z+3 / 2$; (ii) $-x-1 / 2,-y+1, z+1 / 2$; (iii) $x-1, y, z$; (iv) $-x+1 / 2,-y+1, z+1 / 2$; (v) $x-1 / 2,-y+3 / 2,-z+1$; (vi) $x+1, y, z$; (vii) $x+1 / 2,-y+3 / 2,-z+1$; (viii) $-x+1 / 2,-y+1, z-1 / 2$; (ix) $-x+1, y-1 / 2,-z+3 / 2$; (x) $x+1 / 2,-y+1 / 2,-z+1$.

Hydrogen-bond geometry $\left(\AA,{ }^{\circ}\right)$

\begin{tabular}{lllll}
\hline$D-\mathrm{H} \cdots A$ & $D-\mathrm{H}$ & $\mathrm{H} \cdots A$ & $D \cdots A$ & $D-\mathrm{H} \cdots A$ \\
\hline $\mathrm{O} 13-\mathrm{H} 13 \cdots \mathrm{O}^{\text {vi }}$ & 0.82 & 2.21 & $2.982(3)$ & 157 \\
$\mathrm{O} 13-\mathrm{H} 13 \cdots \mathrm{O} 10^{\text {vi }}$ & 0.82 & 2.40 & $3.010(3)$ & 132 \\
\hline
\end{tabular}

Symmetry code: (vi) $x+1, y, z$. 